base pairs from one strand to the other reduced the rate of RNA cleavage nearly 100 -fold. The authors conclude that the RNA-RNA helix is necessary, but not sufficient, for effective $5^{\prime}$ splicing and comment that it is very unlikely that the energy differences between two such closely related 9-bp stems could account for the observed rate differences. Instead, they suggest that "the sequence of (these segments) may play a role in positioning the helix via tertiary interactions between exposed groups on bases in (this) and other parts of the RNA catalyst".

The detailed crystal structures of various model DNA helices support this idea and indicate future directions for experimentation. In addition, the exact role of the 9bp Watson-Crick seondary structure needs to be investigated in more detail, using techniques similar to those of Waring et al. to generate mutations which give aberrant cleavage, rather than no cleavage. Although a helix may help to define the splice site, precision may be conferred by other structural features, with the base pairs needed for stability.

The two papers in this issue address additional aspects of $5^{\prime}$ splice-site specifications in Group I introns. One of these, by Garriga, Lambowitz, Inoue and $\mathrm{Cech}$, takes advantage of a previous finding ${ }^{7}$ that certain RNA-catalysed reactions involving the $5^{\prime}$ splice site of the Tetrahmyena rRNA precursor can occur in trans. For example, when the dinucleotide $\mathrm{CU}$ is incubated with the rRNA precursor in the absence of a guanosine cofactor, normal cleavage at the $5^{\prime}$ splice site is not observed; however, the dinucleotide becomes attached to the $3^{\prime}$ (downstream) exon at the exact $3^{\prime}$ splice site. From this work and the studies of Waring et al. ', it appears that the $5^{\prime}$ exon remains bound to the IGS even after cleavage at the $5^{\prime}$ splice site and that the IGS then positions the $3^{\prime}$ splice site for cleavage. Thus, the interaction which forms the 9-bp stem may normally hold the $5^{\prime}$ exon in position to attack the $3^{\prime}$ splice site, but when cleavage at the $5^{\prime}$ splice site $\left(\ldots U_{\uparrow}\right)$ is blocked, the dinucleotide $\mathrm{CU}$ temporarily disrupts the structure around the $5^{\prime}$ splice site and cleaves, adding itself to the $3^{\prime}$ exon. This explanation for the effect of $\mathrm{CU}$ has been tested by Garriga et al. ${ }^{3}$.

The authors took a second Group I intron, which has a different sequence in the helix spanning the $5^{\prime}$ splice site. In agreement with earlier predictions of Davies et al. ${ }^{\prime}$, the sequence in the Neurospora mitochondrial cytochrome $b$ intron 1 messenger RNA (CUGGGU, in-stead of the CUCUCU of Tetrahymena) has a complementary region in a segment analogous to the IGS of Tetrahymena. Garriga et al. show that CU promotes cleavage of the Neurospora mRNA precursor to give a $3^{\prime}$ exon with GU attached to its $5^{\prime}$ end. $\mathrm{CU}$ is inactive in this assay. Interestingly,

\title{
Galactic strings are stable
}

Loops of cosmic string may be the seeds of galaxies, as many astrophysicists have suggested, but are they stable and do they last long enough to set galactic formation going? Two theorists from Imperial College, London, have just addressed this key issue, and found that the answer is yes (Copeland, E.J. \& Turok, N. Phys. Letts. B 173, 129; 1986).

Initially the strings emerge from the mesh of vortices presumed present after the "phase transition" or "condensation" that takes place when the universe cools through the grand unification temperature - a temperature above which the strong, weak and electromagnetic forces behave as if they had equal strength. The galactic loops are chopped off the mesh about $10^{8}$ seconds (3 years) after the Big Bang. But the seeding of galactic matter cannot take place before the Universe passes the state of equal matter and radiation density some $10^{11}$ seconds $(3,000$ years $)$ later.

Thus the cosmic loops of self-gravitating string, weighing 1,000 tonnes per Fermi $\left(10^{-13} \mathrm{~cm}\right)$, must last 3,000 years - or the stringy explanation of galactic formation will not work.

Basically, Copeland and Turok set out to show that a complete "seed" loop, which should weigh in at about one-hundredth of a galatic mass and be 30 parsecs long at the seeding time, will not intersect itself on a 3,000-year timescale. Other studies have shown that self-intersecting loops cut themselves in two to form smaller loops,

adding trinucleotides to these reactions gives less clear results, leading the authors to point out that "...although WatsonCrick base pairing with the intron binding sites may be an important determinant of the trans-splicing reaction, additional interactions must also contribute to determining the extent of reaction with trinucleotides. Base stacking or nonWatson-Crick hydrogen bonding might allow certain trinucleotides to interact with the normal binding site. Alternatively, the trinucleotide might interact with other, at present unidentified binding sites within the intron". Again we have here base-pairing rules as part of the explanation but additional structural elements are invoked.

In the other paper in this issue $\mathrm{e}^{2} \mathrm{~J} . \mathrm{W}$. Szostak synthesized in vitro a 'core' of the rRNA intron containing about 250 internal bases but neither of the splice junctions nor either of the two 9-nucleotide RNA segments whose base pairing I have described here as an important element in cleavage at the $5^{\prime}$ splice site. Both these segments are present in a second 'substrate' molecule, a 119-base-long RNA which contains the last 32 nucleotides of the $5^{\prime}$ exon and the first 62 nucleotides of the intron, and thus spans the $5^{\prime}$ splice and if this process continued over a timescale short compared with 3,000 years there would be no loops left of the right size to seed galaxies.

In fact, the spectrum of loops that Copeland and Turok start with at $10^{x}$ seconds has already ironed out simple twisting (as when an elastic band is twisted into a figure eight); the loops they begin with are those left after all initial motions that lead to self intersection have done their work. For the problem in hand, it is necessary to consider perturbations of the remaining strings caused by gravitational effects - reaching sufficiently large amplitudes to cause selfintersection. In principle, cusps can form, for example, that will bite off small loops. Copeland and Turok's calculations show that these effects are negligible over a 3,000 -year period, however, so the galactic loops remain effectively stable.

Do the loops remain in present galaxies? No. They steadily shrink, losing mass as gravitational waves, until they vanish in a puff of super-heavy bosons. Looking out into the Universe from our present vantage point, this process was occurring at a redshift of around 100 . The furthest known objects are something over a redshift of 3. However, the larger and so longer-lived loops that are postulated to cause the formation of galactic clusters should still be around. They should be hiding in the clusters with lengths of a few kiloparsecs, and masses of the order of that of a galaxy.

Robert Walgate

site. Neither core enzyme nor substrate RNA is capable of self-cleavage, but gel electrophoresis shows that the core cleaves the substrate accurately to give the expected $5^{\prime}$ end of the intron sequence. The interactions between the two molecules studied by Szostak cannot be stabilized by Watson-Crick structural interactions involving the IGS.

Furthermore, Szostak finds optimal cleavage under conditions quite different from those originally characterized by Cech and colleagues ${ }^{4}$ for the self-catalysed splicing of the intact Tetrahymena rRNA precursor. Remarkably, the optimal temperature is $58^{\circ} \mathrm{C}$. Szostak concludes "it is likely that the structure of the enzyme is stabilized by numerous tertiary interactions, and that the enzyme in turn stabilizes the structure of the substrate."

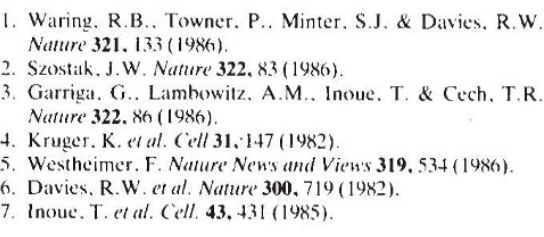

H.D. Robertson is in the Laboratory of Genetics at Rockefeller University, 1230 York Avenue. New York 10021. USA. 